\title{
OPTIMIZATION DESIGN OF MULTISTAGE PUMP IMPELLER BASED ON RESPONSE SURFACE METHODOLOGY
}

\author{
Guangue Peng, Shiming Hong, Hao Chang, Zhuoran Zhang, Fengyi Fan \\ Research Center of Fluid Machinery Engineering and Technology, Jiangsu University, Zhenjiang, China \\ e-mail: pgj@ujs.edu.cn; changhao@ujs.edu.cn
}

\begin{abstract}
The central composite design of the response surface methodology is applied to optimize geometrical parameters of a multistage pump impeller in this paper, and a relevant experiment was conducted. The maximum head difference is $5.6 \%$, and the maximum efficiency difference is $0.73 \%$, which can ensure the accuracy of the investigation. Meanwhile, 30 groups of test schemes are obtained based on the software Design Expert, and the numerical calculation of each scheme is conducted. According to the calculation results and variance analysis, it is found that the effect of response variables of the primary terms blade number, impeller outlet diameter, blade outlet width, and the quadratic terms between the blade number and impeller outlet diameter, blade number and blade wrap angle, impeller outlet diameter and blade outlet width on the head are significant. However, the primary term blade wrap angle, the quadratic terms between the blade number and blade outlet width, impeller outlet diameter and blade wrap angle, blade wrap angle and blade outlet width have no significant effect on the head. Furthermore, a response surface regression model of the single-stage impeller head of a multistage pump was established after removing insignificant factors, and the deviation of the response surface regression model is only $2.4 \%$. The significant sequence of the influence of response variables on the head is the blade number, impeller diameter, blade outlet width, and blade wrap angle. Finally, the optimal geometrical parameters of the impeller are obtained: the number of blades is 6 , the diameter of the impeller is $254 \mathrm{~mm}$, the blade wrap angle is $119^{\circ}$, the outlet width of the blade is $4.3 \mathrm{~mm}$, and the predicted value of the head is $189.19 \mathrm{~m}$. Therefore, the influence rule of impeller geometrical parameters on the head was obtained, which can provide theoretical references for the optimization design of the multistage pump impeller.
\end{abstract}

Keywords: multistage centrifugal pump, optimization design, response surface methodology

\section{Introduction}

Due to the special construction of a multistage centrifugal pump, it can not only play an important role in the industrial production process but can also be widely used in high altitude water supply and many other places. At present, scholars have studied the optimal design of the pump based on a variety of optimization methods. Ping et al. (2021) combined a hybrid algorithm integrated with a machine learning model to improve the isentropic efficiency of a multistage centrifugal pump in an ORC system, and a novel isentropic efficiency prediction model was proposed. Tong et al. (2020) compared the hydraulic loss model and three surrogate models combined with NSGA-pi genetic algorithm respectively. A nonlinear relationship between geometrical parameters and hydraulic performance was obtained. Benturki et al. (2018) selected the efficiency and suction capability of a multistage centrifugal pump as the optimization target, employing the Non-dominated Sorting Genetic Algorithm II (NSGA-II) to enhance volumetric and hydraulic efficiencies. Xu et al. (2021) combined an RBF (Radial Basis Function) neural network model and NSGA-II (Non-Dominated Sorting Genetic Algorithm) optimization algorithm to investigate the influence of geometric parameters of a jet pump on hydraulic performance, and 
the head ratio improved by $30.46 \%$ after the optimization design. Zhang et al. (2021) modified the meridional shape and blade geometry based on the three-dimensional (3D) inverse design method to optimize the efficiency and cavitation performance, and the optimal geometry was obtained by the surrogate model. Shim et al. (2020) analyzed the influence of the blade outlet angle, impeller outlet width and the cross-sectional area of the volute on the operating stability. The objective functions were obtained based on the Latin hypercube sampling. Wang et al. (2020) performed computational studies using one-dimensional theory and the Pareto-based genetic algorithm to improve the energy efficiency of a pump as a turbine. They founded that the blade outlet width, blade exit angles and the blade number had a significant impact on the PAT efficiency. Gan et al. (2020) selected fourteen design variables of the inlet pipe and impeller, and 300 sample cases of the numerical simulation were carried out based on the Latin hypercube sampling (LHS). The results present that the profile of the inlet pipe and the blade plays an important role on the performance of the vertical inline pump. Nitri Asomani et al. (2020) compared the Adaptive Neuro-Fuzzy Inference System (ANFIS), Artificial Neural Network (ANN) and Generalized Regression Neural Network (GRNN) to optimize the geometrical parameter of a pump as a turbine. Xu et al. (2020) conducted numerical studies on the effect of the jet pump geometrical parameter on the efficiency, and the hydraulic performance could be predicted effectively by the Kriging model.

The response surface methodology is an important analysis method, which is widely employed in many engineering fields. Thakkar et al. (2021) selected the blade outlet angle, blade wrap angle and blade outlet width as the optimization variables to improve the hydraulic performance of a sanitary centrifugal pump based on the response surface methodology. The results present that the effect of blade outlet width and blade wrap angle on the efficiency is obvious. Yang et al. (2016) employed the response surface methodology to analyze the relationship between structural parameters of the jet pump and acoustic streaming. The result shows that an increase of the inner diameter can decline the acoustic streaming. Although lots of researchers employ the response surface methodology in fluid machine engineering, few investigations of multistage pump were carried out. Therefore, in this paper, the central composite design of the response surface methodology is applied to optimize geometrical parameters of a multistage pump impeller. Finally, the influence rule of the impeller geometrical parameters on the head was obtained, which can provide theoretical references for the optimization design of the multistage pump impeller.

\section{Calculation method and experimental verification}

\subsection{Control equations and solutions}

In this paper, the continuity equation of the incompressible fluid and the N-S equation were employed. The continuity equation of the incompressible fluid and the N-S equation are applicable to describe the three-dimensional turbulence in a centrifugal pump.

The continuity equation

$$
\frac{\partial\left(\rho u_{i}\right)}{\partial x_{i}}=0
$$

N-S equation for the incompressible fluid

$$
\frac{\partial\left(\rho u_{i}\right)}{\partial t}+\frac{\partial\left(\rho u_{i} u_{j}\right)}{\partial x_{j}}=-\frac{\partial p}{\partial x_{j}}+\rho F_{i}+2 \frac{\partial\left(\mu S_{i j}\right)}{\partial x_{j}}
$$

where $\rho$ - fluid density, $t$ - time, $F_{i}$ - volume force component of the fluid per unit mass, $P$ pressure, $u_{i}, u_{j}(i, j=1,2,3)$ - velocity component of the fluid, $x_{i}, x_{j}(i, j=1,2,3)$ - coordinate component, $\mu$ - molecular viscosity coefficient, $S_{i j}$ - fluid deformation rate tensor 


$$
S_{i j}=\frac{1}{2}\left(\frac{\partial u_{i}}{\partial x_{j}}+\frac{\partial u_{j}}{\partial x_{i}}\right)
$$

In this paper, the Reynolds average method is used to solve the above governing equation, and the two-equation model in the vortex-viscous model is used to close the equations. In addition, the Reynolds number at the inlet of the multistage pump is calculated by

$$
\operatorname{Re}_{\text {inlet }}=\frac{\rho v_{1} d}{\mu}
$$

where $v_{1}$ and $d$ are the velocity and equivalent diameter of the inlet suction pipe, respectively. The value of $\mathrm{Re}_{\text {inlet }}$ is about $10^{5}$ under the design condition. Accordingly, the flow in the multistage pump is totally turbulent and vortical structures should be resolved by an appropriate turbulence model. According to the flow characteristics inside the pump, the standard $k-\varepsilon$ model was selected to predict the separation flow, reflux and a large curvature flow.

The turbulent kinetic energy equation

$$
k=\frac{1}{2} \overline{u_{i}^{\prime} u_{i}^{\prime}}
$$

The dissipation rate equation

$$
\varepsilon=\nu \overline{\left(\frac{\partial u_{i}^{\prime}}{\partial x_{j}}\right)\left(\frac{\partial u_{i}^{\prime}}{\partial x_{j}}\right)}
$$

The transport equation of the viscosity coefficient $\nu_{t}$, turbulent kinetic energy $k$ and dissipation rate $\varepsilon$ in the standard $k-\varepsilon$ model can be expressed as follows

$$
\begin{aligned}
& \nu_{t}=C_{\mu} \frac{k^{2}}{\varepsilon} \\
& \frac{\partial k}{\partial t}+u_{j} \frac{\partial k}{\partial x_{j}}=\frac{\partial}{\partial x_{j}}\left[\left(\nu+\frac{\nu_{t}}{\sigma_{k}}\right) \frac{\partial k}{\partial x_{j}}\right]+G_{k}-\varepsilon \\
& \frac{\partial \varepsilon}{\partial t}+u_{j} \frac{\partial \varepsilon}{\partial x_{j}}=\left[\left(\nu+\frac{\nu_{t}}{\sigma_{\varepsilon}}\right) \frac{\partial \varepsilon}{\partial x_{j}}\right]+C_{\varepsilon 1} \frac{\varepsilon}{k} G_{k}-C_{\varepsilon 2} \frac{\varepsilon^{2}}{k} \\
& G_{k}=\nu_{t}\left(\frac{\partial u_{i}}{\partial x_{j}}+\frac{\partial u_{j}}{\partial x_{i}}\right) \frac{\partial u_{i}}{\partial x_{j}}
\end{aligned}
$$

where $G_{k}$ is the generation term of turbulent kinetic energy $k$ caused by the average velocity gradient, and $\sigma_{k}$ and $\sigma_{\varepsilon}$ are the turbulent Prandtl numbers of turbulent kinetic energy $k$ and dissipation rate $\varepsilon$, respectively. The constants in the transport equation are obtained by best fitting the experimental results of typical flows and the results of calculation examples: $C_{\varepsilon 1}=1.44, C_{\varepsilon 2}=1.92, C_{\mu}=0.09, \sigma_{k}=1.0, \sigma_{\varepsilon}=1.3$.

Coupling of pressure and velocity is realized based on SIMPLEC algorithm (just improving the coefficient during solving the velocity-correction equation). It speeds up convergence and improves calculation efficiency. The governing equation is discretized by the finite volume method. A high resolution discrete scheme was used for the convection term, and the diffusion term adopts the central difference scheme.

\subsection{Calculation model}

In this paper, GRD-100 multistage pump is selected as the research object, and the design parameters are shown in Table 1.

The specific speed can be calculated by the following formula

$$
n_{s}=\frac{3.65 n \sqrt{Q}}{\sqrt[4]{H^{3}}}
$$


Table 1. Design parameters of the multistage pump

\begin{tabular}{|c|c|c|c|c|c|c|c|c|}
\hline $\begin{array}{c}\text { Speed } \\
n \\
{[\mathrm{rpm}]}\end{array}$ & $\begin{array}{c}\text { Flow } \\
Q \\
{\left[\mathrm{~m}^{3} / \mathrm{h}\right]}\end{array}$ & $\begin{array}{c}\text { Head } \\
H \\
{[\mathrm{~m}]}\end{array}$ & $\begin{array}{c}\text { Inlet } \\
\text { diameter } \\
d_{1}[\mathrm{~mm}]\end{array}$ & $\begin{array}{c}\text { Outlet } \\
\text { diameter } \\
d_{2}[\mathrm{~mm}]\end{array}$ & $\begin{array}{c}\text { Impeller } \\
\text { number } \\
Z\end{array}$ & $\begin{array}{c}\text { Impeller } \\
\text { diameter } \\
d_{0}[\mathrm{~mm}]\end{array}$ & Stage & $\begin{array}{c}\text { Specific } \\
\text { speed } \\
n_{s}\end{array}$ \\
\hline " 4600 & 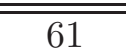 & $17 \overline{1754}$ & $\overline{880}$ & $\overline{\overline{59}}$ & $\overline{66}$ & 252 & $\overline{10}$ & 45.35 \\
\hline
\end{tabular}

The fluid domain model of the multistage pump is simplified into four parts: inlet extension section, impeller, guide vane and outlet ring cavity. UG 3D modeling software is employed to model each part in order to obtain accurate numerical calculation results, the length of the inlet section is extended to 4 times of the inlet diameter, as shown in Fig. 1.

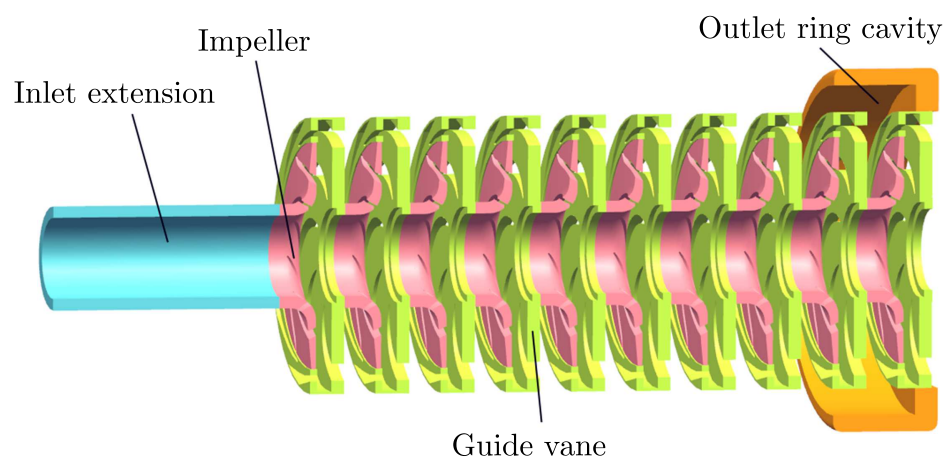

Fig. 1. Multistage pump fluid domain

\subsection{Grid generation}

In this paper, ICEM CFD was used to divide the computing domain into structured grids. The mesh of impeller and guide vane is shown in Fig. 2, and all the grid quality is above 0.3.

(a)

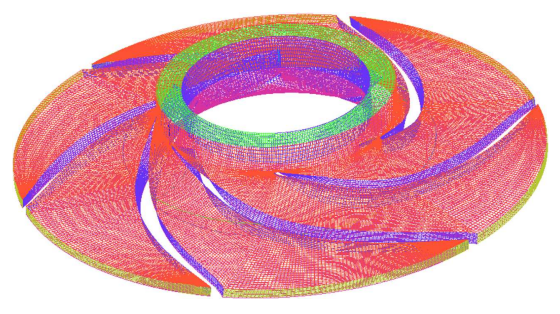

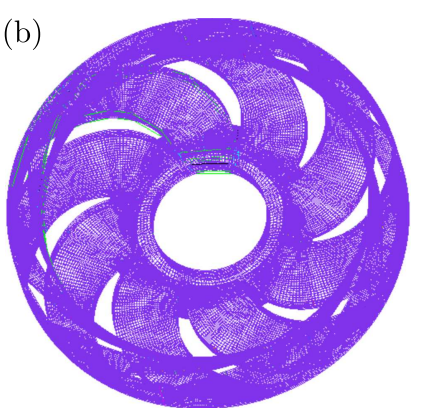

Fig. 2. Grid model: (a) impeller mesh, (b) guide vane mesh

In order to study the effect of the grid number on the calculation results, a grid independence verification was carried out. By calculating and comparing the head and efficiency of four different grids under optimal flow conditions, a reasonable number of grids is finally selected in this paper. It can be seen from Table 2 that with an increase of the grid number, the fluctuation range of the calculation head and efficiency are within $0.3 \%$ and $1.5 \%$, respectively. When the grid number is greater than 7486521 , and the $y^{+}$is less than 50 , the deviation of the head and efficiency is only $4.46 \cdot 10^{-5}$ and $1.36 \cdot 10^{-3}$, considering the consumption of the computation resource and time, the third scheme grid number was selected to carry out the simulation. 
Table 2. Multistage pump performance under different grid numbers

\begin{tabular}{|c|c|c|}
\hline Grid number & Calculation head $H[\mathrm{~m}]$ & Calculation efficiency $\eta[\%]$ \\
\hline \hline 5137862 & 1798.32 & 51.86 \\
\hline 6369034 & 1795.69 & 51.53 \\
\hline 7486521 & 1793.43 & 51.32 \\
\hline 8245953 & 1793.35 & 51.25 \\
\hline
\end{tabular}

\subsection{Boundary conditions}

In this paper, the numerical calculation is employed by commercial software ANSYS CFX. Clean water is selected as the conveying medium. What is more, the calculation domain of the impeller is set as a rotating domain, and the rotating speed is $4600 \mathrm{rpm}$. The inlet, guide vane and outlet ring cavity are set as the static domain. The standard $k-\varepsilon$ model is selected as the turbulence model, and the wall of the domain is set as the non-slip. Meanwhile, the inlet is a total pressure inlet, the outlet is set as a mass flow. The interfaces between the static and rotating domain are selected for data transmission with a frozen rotor model. Considering the rotation speed of the impeller is $4600 \mathrm{rpm}$, the calculation is carried out when the impeller rotates by 3 degrees, and the total calculation period is 8 circles. Therefore, the total time step is 960 steps, the total calculation time is $1.08696 \cdot 10^{-4} \mathrm{~s}$, the physical time scale is adopted as the time control, and the convergence of residual error is set as $10^{-4}$.

\subsection{Experiment verification}

To verify the accuracy of numerical calculation, the test for external characteristics of the multistage pump was carried out on an open experimental bench, as shown in Fig. 3. The experimental bench consists of a multistage pump, motor, pressure transducer torque transducer, speed transducer, and turbine flowmeter. According to the previous research (Peng et al., 2020), measurement deviation is $0.37 \%$, which can satisfy the accuracy of the test analysis. Figure 4 presents the comparison results of the experiment and simulation. Considering that the operating range of the multistage pump is small in the actual operation condition, the numerical simulation of six operation conditions within $0.8 Q \sim 1.3 Q$ was carried out.

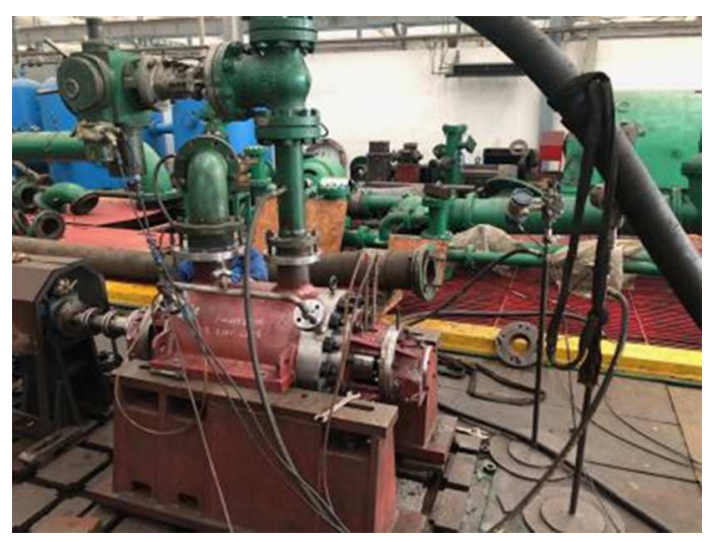

Fig. 3. Experimental bench

It should be stressed that the losses in pumps include hydraulic loss, mechanical loss and volume loss, while the obtained efficiency in simulation is the hydraulic efficiency $\eta_{h}$

$$
\eta=\eta_{h} \eta_{v} \eta_{m} \quad \eta_{h}=\frac{\rho g Q H}{M \omega}
$$




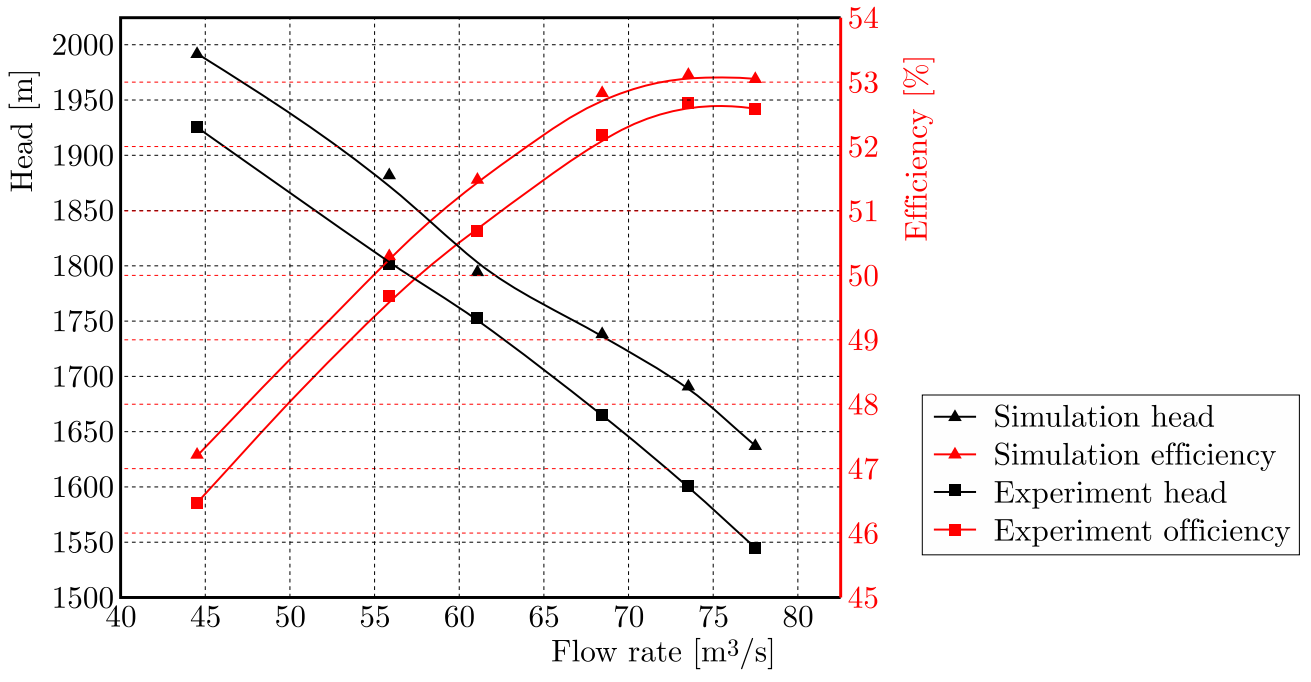

Fig. 4. Comparison of simulation and experiment results

The volume efficiency $\eta_{v}$ and mechanical efficiency $\eta_{m}$ are generally estimated by empirical formulas as follows

$$
\eta_{v}=1-0.028\left(\frac{n_{s}}{100}\right)^{-0.6}=0.955 \quad \eta_{m}=1-0.07\left(\frac{n_{s}}{100}\right)^{1.18}=0.972
$$

It can be seen from Fig. 4 that the numerical calculation results are consistent with the experiment. The maximum head difference is $5.6 \%$, and the maximum efficiency difference is $0.73 \%$. The error of the calculation method is small, which can ensure the accuracy of investigation.

\section{Results and discussions}

\subsection{Response surface methodology}

The response surface methodology is an important analysis method, which is widely employed in many engineering fields. Therefore, in this paper, the central composite design of the response surface methodology is applied to optimize geometrical parameters of the multistage pump impeller. The head of the single-stage impeller is selected as the target variable $y$, and the number of impeller blades $z$, impeller outlet diameter $D_{\text {out }}$, blade wrap angle $\varphi$, and blade outlet width $b$ are set as the response variables, which are expressed as $x_{1}, x_{2}, x_{3}, x_{4}$, respectively. The geometrical parameters of the impeller are shown in Table 3.

Table 3. Design parameters of the original pump impeller

\begin{tabular}{|c|c|c|c|}
\hline$z$ & $D_{\text {out }}[\mathrm{mm}]$ & $\varphi\left[^{\circ}\right]$ & $b[\mathrm{~mm}]$ \\
\hline \hline 6 & 252 & 115 & 5 \\
\hline
\end{tabular}

The range of the impeller parameters $x_{1}, x_{2}, x_{3}$, and $x_{4}$ is presented in Table 4 .

In this paper, the optimization design method is carried out to obtain the maximum value of the target head variable by employing the software of Design Expert, and the number of response variables involved in the experiment is 4 . In order to decline the deviation between different variables, the standardized processing of each response variable range is carried out, according to the quadratic general rotational design method, as shown in Table 5. 
Table 4. Range of $x_{1}, x_{2}, x_{3}, x_{4}$

\begin{tabular}{|c|c|c|c|c|}
\hline$x$ & $\begin{array}{c}\text { Minimim } \\
\text { value }\end{array}$ & $\begin{array}{c}\text { Maximum } \\
\text { value }\end{array}$ & $\begin{array}{c}\text { Average } \\
\text { value }\end{array}$ & $\begin{array}{c}\text { Standard } \\
\text { deviation }\end{array}$ \\
\hline \hline$x_{1}$ & 4 & 6 & 5 & 1 \\
\hline$x_{2}$ & 245 & 255 & 250 & 5 \\
\hline$x_{3}$ & 110 & 120 & 115 & 5 \\
\hline$x_{4}$ & 4 & 6 & 5 & 1 \\
\hline
\end{tabular}

Table 5. Design parameters of the coding table

\begin{tabular}{|l|c|c|c|c|c|c|c|c|}
\hline \multirow{2}{*}{\multicolumn{1}{|c|}{$x$ range }} & \multicolumn{4}{|c|}{ Actual variable $x$} & \multicolumn{5}{c|}{ After standardization $z$} \\
\cline { 2 - 10 } & $x_{1}$ & $x_{2}$ & $x_{3}$ & $x_{4}$ & $z_{1}$ & $z_{2}$ & $z_{3}$ & $z_{4}$ \\
\hline \hline Upper level & 4 & 245 & 110 & 4 & 1 & 1 & 1 & 1 \\
\hline Zero level & 5 & 250 & 115 & 5 & 0 & 0 & 0 & 0 \\
\hline Lower level & 6 & 255 & 120 & 6 & -1 & -1 & -1 & -1 \\
\hline Upper point from zero level & 7 & 260 & 125 & 7 & 2 & 2 & 2 & 2 \\
\hline Lower point from zero level & 3 & 240 & 105 & 3 & -2 & -2 & -2 & -2 \\
\hline Standard deviation & 1 & 5 & 5 & 1 & & & & \\
\hline
\end{tabular}

According to the software of Design Expert, 30 groups of test schemes are obtained, and the numerical calculation of each scheme is conducted. The regression equation is supposed as follows

$$
\begin{aligned}
E y & =f\left(z_{1}, z_{2}, z_{3}, z_{4}\right)=b_{0}+b_{1} z_{1}+b_{2} z_{2}+b_{3} z_{3}+b_{4} z_{4}+b_{11} z_{1}^{2}+b_{22} z_{2}^{2} \\
& +b_{33} z_{3}^{2}+b_{44} z_{4}^{2}+b_{12} z_{1} z_{2}+b_{23} z_{2} z_{3}+b_{34} z_{3} z_{4}
\end{aligned}
$$

The test was carried out according to the test points of the general rotating combination design in Table 6 , where $A=z_{1}, B=z_{2}, C=z_{3}$, and $D=z_{4}$. The $y$ value of the test data for each group was filled into the corresponding table and calculated after modeling the impeller design parameters of each test point. The impeller models of scheme 4 , scheme 13 , and scheme 15 are shown in Fig. 5.
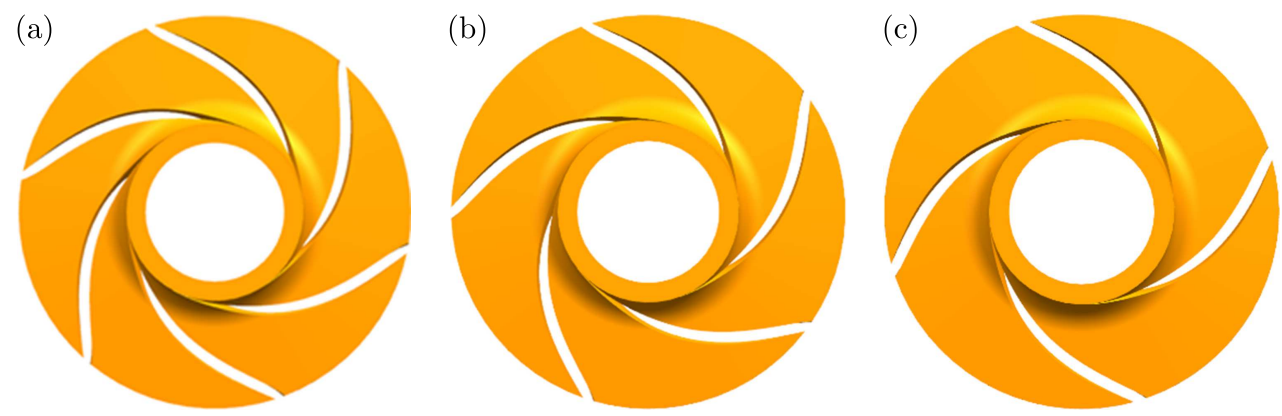

Fig. 5. Impeller model diagram: (a) scheme 4, (b) scheme 13, (c) scheme 15

\subsection{Analysis of the variance and response regression model}

Variance as an evaluation variable can be used to indicate the degree of dispersion between a random variable and the expected value. The mathematical description of variance is the average value of the square of the difference between each data and its respective average value. The variance of the target variable head is obtained as shown in Table 7 . 
Table 6. Test schemes

\begin{tabular}{|c|c|c|c|c|c|c|}
\hline $\begin{array}{c}\text { Standard } \\
\text { order }\end{array}$ & $\begin{array}{l}\text { Run } \\
\text { order }\end{array}$ & $\begin{array}{c}\text { Factor } 1 \\
z_{1} \text { impeller } \\
\text { number }\end{array}$ & $\begin{array}{c}\text { Factor } 2 \\
z_{2} \text { impeller } \\
\text { diameter }\end{array}$ & $\begin{array}{c}\text { Factor } 3 \\
z_{3} \text { wrap } \\
\text { angle }\end{array}$ & $\begin{array}{c}\text { Factor } 4 \\
z_{4} \text { outlet } \\
\text { width }\end{array}$ & $\begin{array}{c}\text { Response } \\
\text { head } y\end{array}$ \\
\hline 17 & 1 & -2 & 0 & 0 & $\overline{0}$ & $\begin{array}{l}162.80 \\
\end{array}$ \\
\hline 8 & 2 & -1 & -1 & -1 & 1 & 194.82 \\
\hline 14 & 3 & -1 & 1 & $\overline{-1}$ & $\overline{-1}$ & 187.22 \\
\hline 12 & 4 & -1 & -1 & 1 & -1 & 199.06 \\
\hline 5 & 5 & 1 & 1 & -1 & 1 & 152.91 \\
\hline 27 & 6 & 0 & 0 & 0 & 0 & 117.78 \\
\hline 7 & 7 & 1 & -1 & -1 & 1 & 180.11 \\
\hline 25 & 8 & 0 & 0 & 0 & 0 & 177.78 \\
\hline 19 & 9 & 0 & -2 & 0 & 0 & 180.16 \\
\hline 10 & 10 & -1 & 1 & 1 & -1 & 183.79 \\
\hline 21 & 11 & 0 & 0 & -2 & 0 & 183.22 \\
\hline 22 & 12 & 0 & 0 & 2 & 0 & 180.49 \\
\hline 29 & 13 & 0 & 0 & 0 & 0 & 177.78 \\
\hline 30 & 14 & 0 & 0 & 0 & 0 & 177.78 \\
\hline 13 & 15 & 1 & 1 & -1 & -1 & 154.05 \\
\hline 4 & 16 & -1 & -1 & 1 & 1 & 197.56 \\
\hline 1 & 17 & 1 & 1 & 1 & 1 & 162.05 \\
\hline 23 & 18 & 0 & 0 & 0 & -2 & 194.57 \\
\hline 26 & 19 & 0 & 0 & 0 & 0 & 177.78 \\
\hline 11 & 20 & 1 & -1 & 1 & -1 & 169.52 \\
\hline 15 & 21 & 1 & -1 & -1 & -1 & 184.15 \\
\hline 6 & 22 & -1 & 1 & $\overline{-1}$ & 1 & 186.64 \\
\hline 3 & 23 & 1 & $\overline{-1}$ & 1 & 1 & 163.17 \\
\hline 2 & 24 & -1 & 1 & 1 & 1 & 182.43 \\
\hline 28 & 25 & 0 & 0 & 0 & 0 & 177.78 \\
\hline 20 & 26 & 0 & 2 & 0 & 0 & 175.40 \\
\hline 18 & 27 & 2 & 0 & 0 & 0 & 206.57 \\
\hline 24 & 28 & 0 & 0 & 0 & 2 & 189.29 \\
\hline 9 & 29 & 1 & 1 & 1 & -1 & 154.91 \\
\hline 16 & 30 & -1 & -1 & -1 & -1 & 196.75 \\
\hline
\end{tabular}

It can be seen from Table 7 that the model significance test is $p<0.05$, indicating that the model is statistically significant. The effect of response variables of the primary terms $A, B, D$, the quadratic terms $A B, A C, B D, B^{2}$ on the head are significant $(p<0.05)$. However, the primary term $C$, the quadratic terms $A D, B C, C D, A^{2}, C^{2}$ and $D^{2}$ have no significant effect on $y$, which can be eliminated in the calculation.

The response surface is an approximate functional relationship obtained by regression fitting of a large amount of experimental data. It can be expressed as a functional formula of the target variable $y$ and a set of response variables $\left(x_{1}, x_{2}, \ldots\right)$

$$
y=f\left(x_{1}, x_{2}, \ldots\right)
$$

When there is only one variable, the response surface appears as a straight line. In general, there are two variables, which appear as a curved surface in space. The surface equation has also the form of a regression equation 
Table 7. Analysis of the variance table

\begin{tabular}{|l|c|c|c|c|c|}
\hline \multicolumn{1}{|c|}{$\begin{array}{c}\text { Source of } \\
\text { variation }\end{array}$} & $\begin{array}{c}\text { Sum of } \\
\text { squares }\end{array}$ & $d f$ & $\begin{array}{c}\text { Mean } \\
\text { square }\end{array}$ & $F$ value & $P$ value \\
\hline \hline Model & 4532.15 & 14 & 323.73 & 5.50 & 0.0002 \\
\hline$A$ blades & 3624.57 & 1 & 3624.57 & 61.60 & $<0.0001$ \\
\hline$B$ diameter & 519.13 & 1 & 519.13 & 8.82 & 0.0095 \\
\hline$C$ wrap angle & 109.09 & 1 & 109.09 & 3.69 & 0.0609 \\
\hline$D$ outlet width & 135.43 & 1 & 135.43 & 7.62 & 0.0389 \\
\hline$A B$ & 52.89 & 1 & 52.89 & 2.53 & 0.0146 \\
\hline$A C$ & 46.32 & 1 & 46.32 & 2.21 & 0.0171 \\
\hline$A D$ & 26.81 & 1 & 26.81 & 1.13 & 0.0974 \\
\hline$B C$ & 10.06 & 1 & 10.06 & 0.4803 & 0.0505 \\
\hline$B D$ & 13.02 & 1 & 13.02 & 0.6217 & 0.0450 \\
\hline$C D$ & 19.70 & 1 & 19.70 & 0.3351 & 0.0857 \\
\hline$A^{2}$ & 7.41 & 1 & 7.41 & 0.3540 & 0.0566 \\
\hline$B^{2}$ & 13.02 & 1 & 13.02 & 0.6217 & 0.0450 \\
\hline$C^{2}$ & 3.66 & 1 & 3.66 & 0.1747 & 0.0685 \\
\hline$D^{2}$ & 1.76 & 1 & 1.76 & 0.1253 & 0.1035 \\
\hline \hline Residual & 882.67 & 15 & 58.84 & & \\
\hline Loss of quasi item & 882.67 & 10 & 88.27 & & \\
\hline Pure error & 0.0000 & 5 & 0.0000 & & \\
\hline Sum & 5414.82 & 29 & & & \\
\hline
\end{tabular}

$$
y=a_{1}+a_{2} \sum_{i=1}^{n} x_{i}+a_{3} \sum_{i=1}^{n} x_{i}^{2}+a_{4} \sum_{i=1}^{n} x_{i} x_{j}
$$

In the formula, $x_{i}$ represents the primary response variable, $x_{i}^{2}$ represents the quadratic response variable, and $x_{i} x_{j}$ represents the bivariate interaction term. After removing the insignificant factors, the response surface regression model of the single-stage impeller head of the multistage pump should be

$$
H=177.78-8.29 A+4.65 B-0.0333 D+2.57 A B-2.41 A C+1.12 B D+2.18 B^{2}
$$

To verify the accuracy of the response surface regression model, the original pump impeller design parameters are added in the regression model. The predicted value of the single-stage impeller head is $185.59 \mathrm{~m}$, while the numerical simulation result of the original pump single-stage impeller head is $181.31 \mathrm{~m}$. The deviation of the response surface regression model is only $2.4 \%$. Therefore, the predicted result is more reliable, indicating that the regression model of the single-stage impeller head of the multistage pump based on the response surface method is considered feasible.

\subsection{Response surface analysis}

Figures 6 to 11 present quadratic term interaction diagrams between the target variable single-stage impeller head $H$ and the response variable blade number $z$, impeller diameter $D_{\text {out }}$, blade wrap angle $\varphi$ and blade outlet width $b$.

Figure 6a shows the contour distribution of the quadratic term interaction diagram of the impeller diameter and blade number. Figure $6 \mathrm{~b}$ presents the response surface distribution. The contour distribution is the projection of the response surface distribution on the horizontal plane, which represents the interaction between two independent variables, and other variables remain 
at zero levels. It can be seen from Fig. 6 that within the range of the number of blades $(4,6)$ and the diameter of the impeller $(245,255)$, the influence of interaction between the blade number and diameter of the impeller on the head represents a positive correlation. When the number of blades is constant, with the improvement of impeller diameter, the head presents an increasing trend. At the same time, when the impeller diameter is constant, the number of blades and the head also show a positive correlation.

(a)

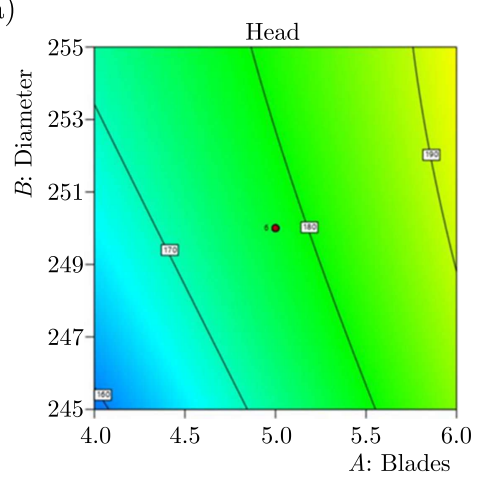

(b)

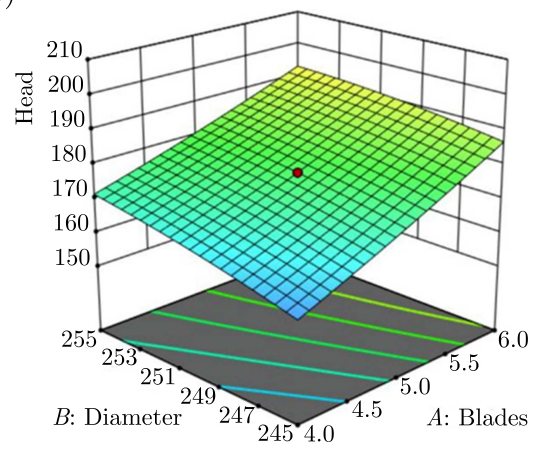

Fig. 6. Quadratic term interaction diagram of the impeller diameter and blade number: (a) contour distribution, (b) response surface distribution

Figure 7 shows the influence of the quadratic term interaction between the blade number and the blade wrap angle on the head, when the blade wrap angle is constant. The head is close to linear growth as the blade number increases. However, when the number of blades is constant within the range of $(4,4.5)$, the head increases slowly as the blade wrap angle improves. But in the range of $(5,5.5)$, with the improvement of the wrap angle, the head remains without any change, and the contour line is a vertical line, which indicates that the blade wrap angle has no effect on the head in this range. Furthermore, in the range of $(5.5,6)$, with an increase of the wrap angle, the head presents a decreasing trend, which shows a negative growth status.

(a)

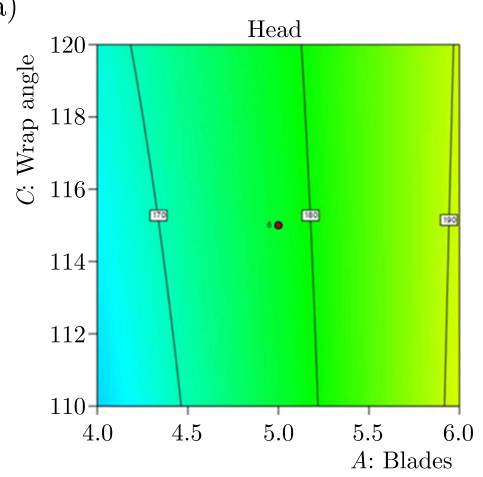

(b)

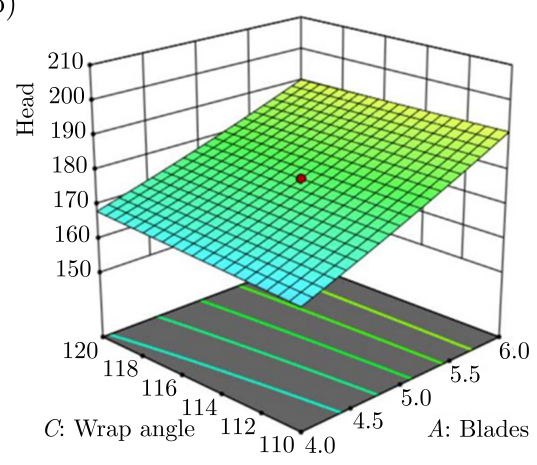

Fig. 7. The quadratic term interaction diagram of the impeller wrap angle and blade number: (a) contour distribution, (b) response surface distribution

In Fig. 8, the response surface distribution of the blade outlet width and blade number on the head shows a downwardly concave shape of the space surface, and the contour distribution presents a parabolic figure that opens to the left. When the outlet width is constant, the head will increase with the improvement of the blade number. What is more, when the number of blades is constant, with the increasing blade outlet width, the head decreases firstly and then increases, which indicates that there is a minimum of the head in the range of $(4,6)$. 
(a)

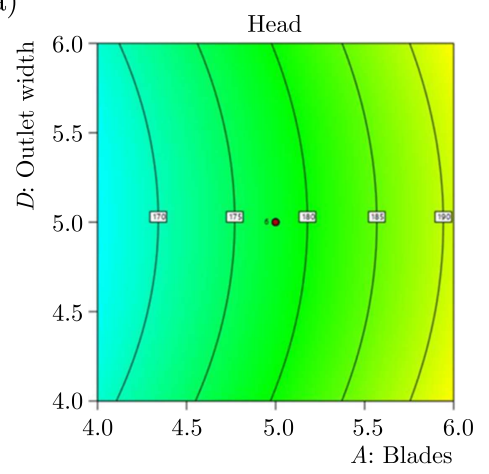

(b)

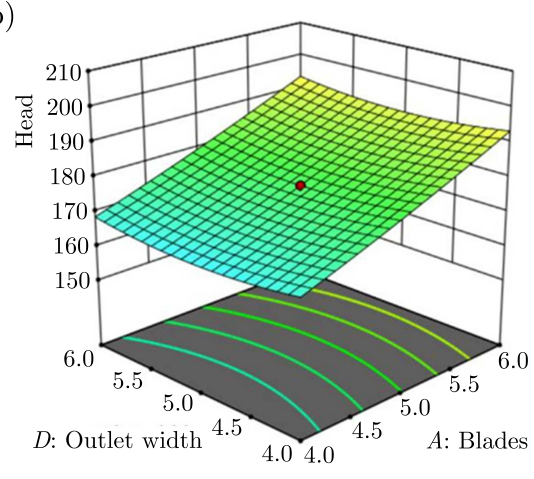

Fig. 8. Quadratic term interaction diagram of the blade outlet width and blade number: (a) contour distribution, (b) response surface distribution

According to Fig. 9, it can be found that within the range of the impeller diameter $(245,255)$ and the blade wrap angle $(110,120)$, the response surface is almost flat. When the blade wrap angle is constant, the relationship between the impeller diameter and the head shows a positive correlation. When the impeller diameter is constant, the variation of the blade wrap angle has no significant effect on the head. Especially in the range of $(247,249)$, the contour line is vertical, indicating that the influence of the blade wrap angle on the head is slight. At the same time, the influence of the quadratic term interaction relationship between the impeller diameter and the blade wrap angle on the head is not obvious, which can also prove that the quadratic term $B C$ has no significant effect on the head in the variance analysis.

(a)

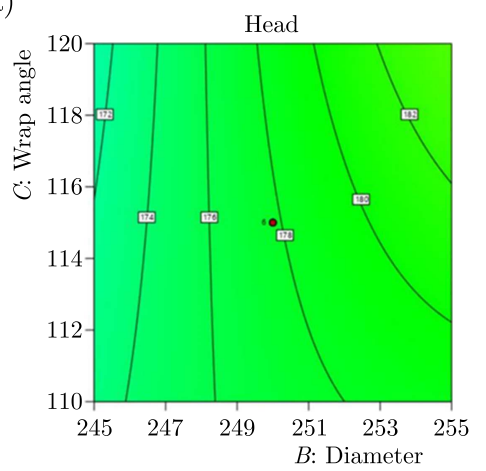

(b)

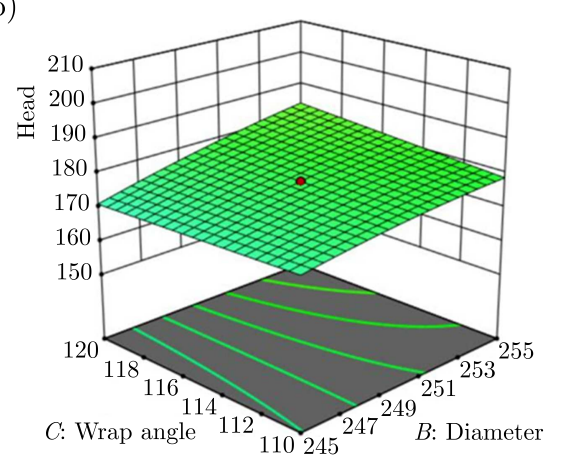

Fig. 9. Quadratic term interaction diagram of the impeller diameter and blade wrap angle: (a) contour distribution, (b) response surface distribution

In Fig. 10, the response surface distribution of the blade outlet width and blade number on the head shows a downwardly concave shape of the space surface, and the contour distribution presents a parabolic figure that opens to the right. That is, when the blade outlet width is constant, as the impeller diameter increases, the head gradually improves. However, when the number of blades is constant, with an increase of the blade outlet width, the head decreases firstly and then increases, which indicates that there is a minimum of the head in the range of $(4,6)$.

It can be seen from Fig. 11 that within the range of the blade wrap angle $(110,120)$ and blade outlet width $(4,6)$, the shape of the response surface distribution is similar to Fig. 10, but its overall slope is smaller and closer to the plane, which can also prove that the influence of the interaction term between the blade wrap angle and blade outlet width is not significant in the analysis of variance. When the blade outlet width is constant, with an increase of the blade 
(a)

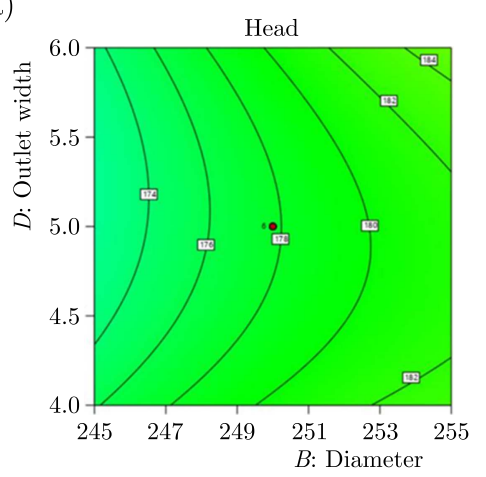

(b)

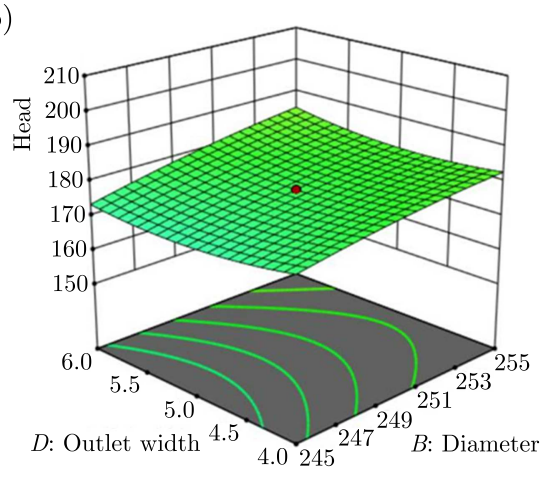

Fig. 10. Quadratic term interaction diagram of the impeller diameter and blade outlet width: (a) contour distribution, (b) response surface distribution

(a)

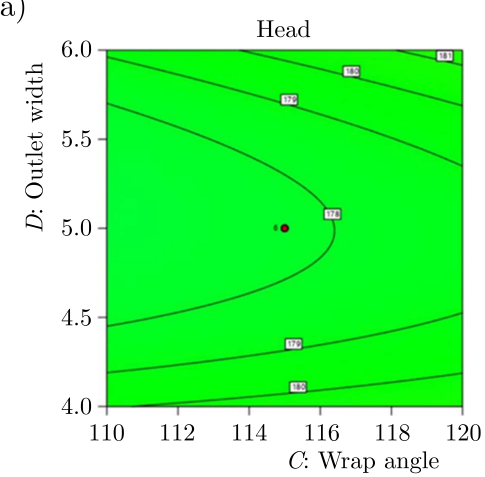

(b)

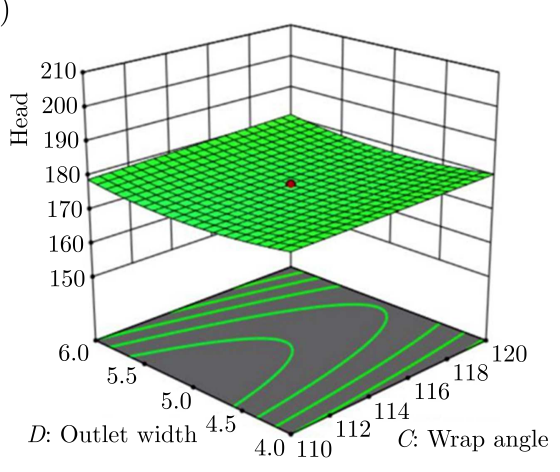

Fig. 11. Double-factor interaction diagram of the blade wrap angle and blade outlet width: (a) contour distribution, (b) response surface distribution

wrap angle, the head continues to improve. Furthermore, when the blade wrap angle is constant as the blade outlet width increases, the head decreases firstly and then increases.

According to the above analysis of the response surface distribution, it can be seen that the quadratic term interaction terms between the blade number and impeller diameter have the most significant impact on the head, while the influence of interaction terms between the blade wrap angle and blade outlet width on the head is not obvious. Combining with the analysis of the variance table, in the primary item, the $P$ value of the blade number is the smallest, which indicates that the number of the blades has an extremely significant impact on the head. Therefore, the significant sequence of the influence of response variables on the head is blade number, impeller diameter, blade outlet width, and blade wrap angle.

Among the quadratic term interaction terms, the order of significance of the influencing factors is consistent with that of the response surface map. The quadratic terms $A D, B C, C D$, $A^{2}, C^{2}$ and $D^{2}$ have no significant effect on the head, the significant sequence of the influence of response variables on the head is $A B, A C, B D$ and $B^{2}$, which verifies that the analysis results of the response surface map are correct and effective.

Figure 12 shows a good agreement between the actual and predicted values of the response. It can be seen from the regression model of the head that the coefficient before the quadratic term is positive, indicating that the solution has a maximum value in the interval. By solving the equation, in the case of increasing the head as much as possible, the four design parameters of the optimized impeller and the predicted value of the single-stage head can be obtained: the number of blades is 6 , diameter of the impeller is $254 \mathrm{~mm}$, the blade wrap angle is $119^{\circ}$, width of the blade outlet is $4.3 \mathrm{~mm}$ and the predicted head value is $189.19 \mathrm{~m}$. 


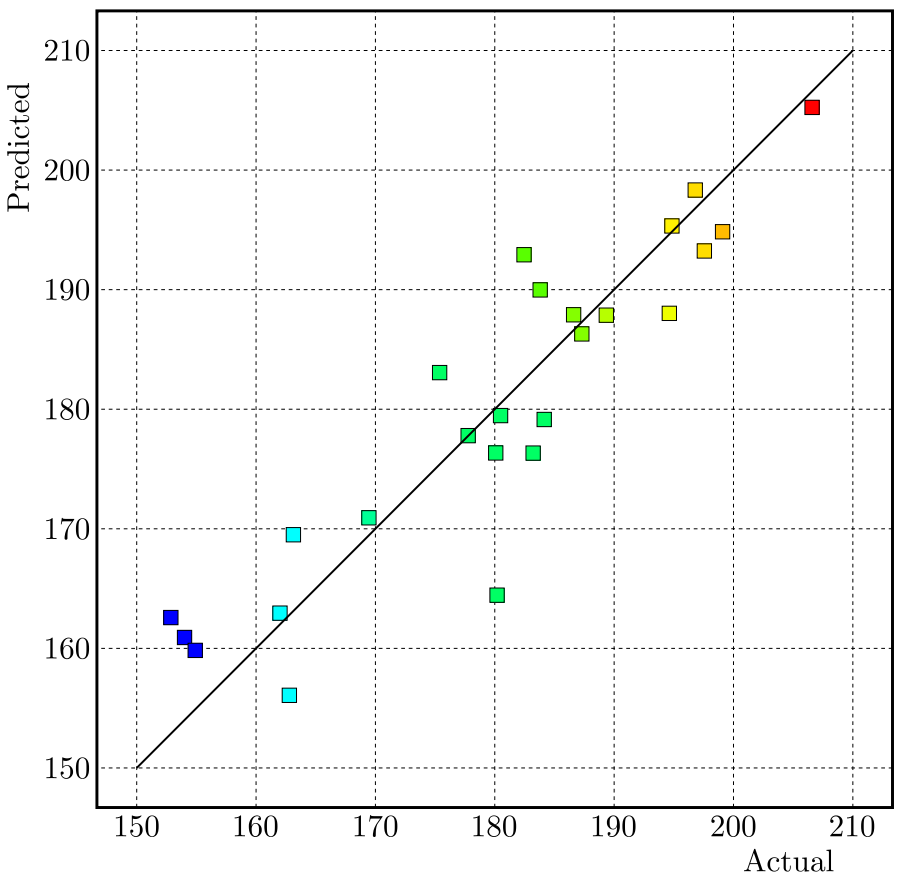

Fig. 12. Distribution of the predicted and actual values

\section{Conclusions}

In this paper, the central composite design of the response surface methodology is applied to optimize the geometry parameters of a multistage pump impeller, and an experiment was conducted. The maximum head difference is $5.6 \%$, and the maximum efficiency difference is $0.73 \%$. The error of the calculation method is small, which can ensure the accuracy of investigation. Therefore, the conclusions are drawn as follows:

- 30 groups of test schemes are obtained based on the software Design Expert, and numerical calculation of each scheme is conducted. According to the calculation results and variance analysis, it is found that the effect of response variables of the primary terms number of the impeller blades, impeller outlet diameter, blade outlet width, and the quadratic terms between the impeller blade number and impeller outlet diameter, impeller blades number and blade wrap angle, impeller outlet diameter and blade outlet width on the head are significant. However, the primary term blade wrap angle, quadratic terms between the impeller blade number and blade outlet width, impeller outlet diameter and blade wrap angle, blade wrap angle and blade outlet width have no significant effect on the head.

- The response surface regression model of the single-stage impeller head of the multistage pump was established after removing the insignificant factors. The regression model equation is $H=177.78-8.29 A+4.65 B-0.0333 D+2.57 A B-2.41 A C+1.12 B D+2.18 B^{2}$. Meanwhile, the original pump impeller design parameters are added in the regression model, and the predicted value of the single-stage impeller head is $185.59 \mathrm{~m}$, the deviation of the response surface regression model is only $2.4 \%$. Therefore, the predicted result is more reliable, the regression model of the single-stage impeller head of the multistage pump based on the response surface method is considered feasible.

- According to the analysis of the response surface distribution, it can be seen that the quadratic term interaction terms between the blade number and impeller diameter have the most significant impact on the head, while the influence of interaction terms between the blade wrap angle and blade outlet width on the head is not obvious. Furthermore, the 
significant sequence of the influence of response variables on the head is the blade number-impeller diameter-blade outlet width-blade wrap angle. Finally, the optimal geometrical parameters of the impeller are obtained: the number of blades is 6 , diameter of the impeller is $254 \mathrm{~mm}$, the blade wrap angle is $119^{\circ}$, outlet width of the blade is $4.3 \mathrm{~mm}$ and the predicted value of the head is $189.19 \mathrm{~m}$.

\section{Acknowledgments}

The authors gratefully acknowledge the support from Open Research Fund Program of State key Laboratory of Hydroscience and Engineering grant number: sklhse-2020-E-01; Open Research Subject of Key Laboratory of Fluid Machinery and Engineering (Xihua University) grant number LTJX2021-003; Open Research Subject of Hubei Key Laboratory of Hydroelectric Machinery Design and Maintenance (China Three Gorges University) grant number 2020KJX07; The 69th batch of general funding from the China Postdoctoral Science Foundation grant number: 2021M691298; Priority Academic Program Development of Jiangsu Higher Education Institutions (PAPD).

\section{References}

1. Benturki M., Dizene R., Ghenaiet A., 2018, Multi-objective optimization of two-stage centrifugal pump using NSGA-II algorithm, Journal of Applied Fluid Mechanics, 11, 4, 929-942

2. Gan X., Pei J., Wang W., Yuan S., Tang Y., 2020, Multi-component optimization of a vertical inline pump based on multi-objective pso and artificial neural network, Journal of Mechanical Science and Technology, 34, 12, 4883-4896

3. Nitri Asomani S., Yuan J., Wang L., Appiah D., Adu-Poku K.A., 2020, The impact of surrogate models on the multi-objective optimization of Pump-As-Turbine (PAT), Energies, 13, 9, 2271

4. Peng G., Hunng X., Zhou L., Zhou G., Zhou H., 2020, Solid-liquid two-phase flow and wear analysis in a large-scale centrifugal slurry pump, Engineering Failure Analysis, 114, 104602

5. Ping X., Yang F., Zhang H., Zhang J., Zhang W., Song G., 2021, Introducing machine learning and hybrid algorithm for prediction and optimization of multistage centrifugal pump in an ORC system, Energy, 222, 120007

6. Shim H.S., Kim K.Y., 2020, Design optimization of the impeller and volute of a centrifugal pump to improve the hydraulic performance and flow stability, Journal of Fluids Engineering, 142, 10

7. Thakkar S., Vala H., Patel V., Patel R., 2021, Performance improvement of the sanitary centrifugal pump through an integrated approach based on response surface methodology, multi-objective optimization and CFD, Journal of the Brazilian Society of Mechanical Sciences and Engineering, 43, 1, 1-15

8. Tong S., Zhao H., Liu H., Yu Y., Li J., Cong F., 2020, Multi-objective optimization of multistage centrifugal pump based on surrogate model, Journal of Fluids Engineering, 42, 1, 011101

9. Wang L., Nitri Asomani S., Yuan J., Appiah D., 2020, Geometrical optimization of pump-as-turbine (PAT) impellers for enhancing energy efficiency with 1-D theory, Energies, 13, 16, 4120

10. Xu K., Wang G., Wang L., Yun F., Sun W., Wang K., Chen X., 2020, Parameter analysis and optimization of annular jet pump based on Kriging model, Applied Sciences, 10, 21, 7860

11. Xu K., Wang G., Zhang L., Wang L., Yu F., Sun W., Wang K., Chen X., 2021, Multi-objective optimization of jet pump based on RBF neural network model, Journal of Marine Science and Engineering, 9, 2, 236 
12. Yang P., Liu Y., Zhong G.-Y., 2016, Prediction and parametric analysis of acoustic streaming in a thermoacoustic Stirling heat engine with a jet pump using response surface methodology, Applied Thermal Engineering, 103, 1004-1013

13. Zhang L., Davila G., Zangeneh M., 2021, Multi-objective optimization of a high specific speed centrifugal volute pump using three-dimensional inverse design coupled with computational fluid dynamics simulations, Journal of Fluids Engineering, 143, 2

Manuscript received June 16, 2021; accepted for print August 25, 2021 